Neurosurg Focus 20 (2):E7, 2006

\title{
Odontoid synchondrosis fractures in children
}

\author{
Daniel R. Fassett, M.D., M.B.A., Todd MCCall, M.D., and Douglas L. BrockMeYer, M.D. \\ Department of Neurosurgery, Primary Children's Medical Center, University of Utah, \\ Salt Lake City, Utah
}

\begin{abstract}
Object. Odontoid synchondrosis fractures, although rare in the overall incidence of spinal trauma, are one of the more common fractures in young children. The goal of this study was to evaluate the demographic data, incidence of neurological deficits, treatment strategies, and outcomes in a combined series of odontoid synchondrosis fractures treated at the authors' institution and reported in other series.

Methods. In a retrospective chart review, the authors identified four odontoid synchondrosis fractures treated at their hospital since January 2000; these were combined with cases reported in six other series in the literature, yielding a total of 55 patients. Data regarding the patients' age, sex, delayed diagnosis, odontoid displacement, neurological deficits, treatment, and fusion status were collected.

The patients' ages ranged from 9 months to 7 years (mean 2.8 years), with neither sex predominating. Diagnosis was delayed in eight cases. The orientation of the odontoid fracture was reported for 36 patients, with $94 \%$ experiencing anterior displacement. Spinal cord injury (SCI) was noted in 15 patients, including 11 with complete injuries and eight with SCI at the cervicothoracic junction. Forty-two (93\%) of 45 patients with fractures initially treated with external immobilization attained fusion. Eight patients were treated with surgery; four initially, with no attempt at conservative therapy, three after failed halo immobilization, and one after nonunion because of delayed diagnosis.

Conclusions. Odontoid synchondrosis fractures can be difficult to diagnose. In children younger than 7 years of age who present with neck pain or neurological deficits attributable to SCI, this fracture should be suspected. Given the high rate of fusion attained with conservative therapy, it is recommended for most synchondrosis fractures, although surgery may be warranted for individual cases.
\end{abstract}

KEY WORDS - odontoid fracture • synchondrosis • trauma • axis • children

$\mathrm{T}$ RAUMATIC cervical SCIs in the upper cervical spine are more common in young children than in adults, in whom most injuries involve the lower cervical spine. ${ }^{11}$ The $\mathrm{C}-2$ is the most commonly injured vertebra in children, and odontoid synchondrosis fractures are among the more common cervical spine fractures in patients younger than 7 years of age. ${ }^{10}$

In young children, the axis is divided by synchondroses between the dens, body, and neural arches (Fig. 1). The cartilaginous plate between the dens and the body of C-2 is an area of potential weakness that does not ossify until a child is 5 to 7 years old. ${ }^{4,10}$ The weak synchondrosis, in combination with the relatively large size of a child's head, which can act as a pendulum in high-speed trauma, makes the odontoid synchondrosis prone to traumatic injury.

\section{Clinical Material and Methods}

A retrospective chart review was performed using the surgical database at Primary Children's Medical Center to identify odontoid synchondrosis fractures treated since

Abbreviations used in this paper: $\mathrm{CT}=$ computerized tomography; $\mathrm{MR}=$ magnetic resonance; $\mathrm{SCI}=$ spinal cord injury.
January 1,2000 . In this search we found four patients, who were combined with patients from six other reported series in the literature (Table 1)..$^{1,3,6-8,10}$ Data regarding age, sex, fracture displacement/angulation, delay in diagnosis, neurological deficits, treatment strategy, and outcome were collected from all series.

\section{Results}

In this retrospective review, we found four patients who were treated for odontoid synchondrosis fractures at our institution between January 2000 and December 2004. These patients were combined with 51 cases from other series reported in the literature. The patients' ages ranged from 9 months to 7 years, with a mean age of 2.8 years. Sex was reported for 50 patients ( 27 girls and 23 boys).

Neurological deficits consistent with SCI were noted in 15 patients, 11 of whom had complete injuries. In eight of the SCIs, the level of the injury was noted to be at the cervicothoracic junction, based on clinical findings or MR imaging. Among the 36 patients in whom fracture orientation was noted, 34 had anterior displacement or angulation, and one patient each had posterior displacement and neutral alignment.

Initial treatment was reported for 52 of the 55 patients 
A

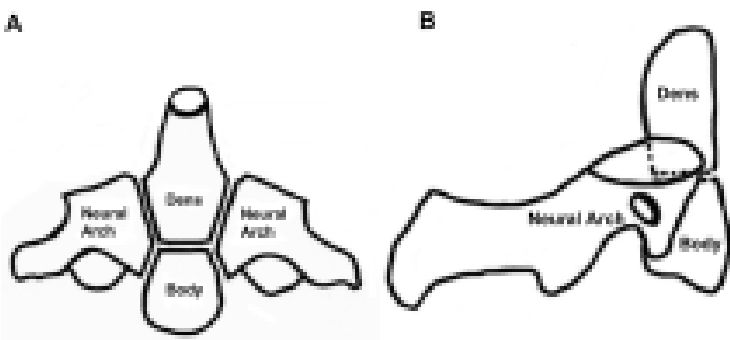

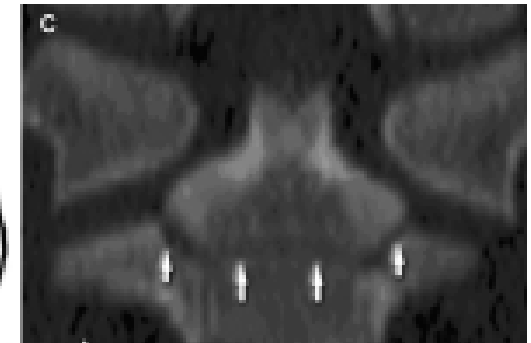

FIG. 1. The axis is formed from four primary ossification centers: the body, the two neural arches, and the dens. These ossification centers are separated by a cartilaginous plate called a synchondrosis. The synchondrosis between the dens and the body of C-2 is prone to fractures in young children. Ventral (A) and lateral (B) drawings depicting the synchondrosis of C-2. Anteroposterior reconstructed CT scan (C) demonstrating the synchondrosis (arrows) in a 3-year-old boy.

(Table 2), and consisted of external stabilization in 45 (Minerva orthosis in 20 patients, halo immobilization in 20 , cervical traction in three, and cervical collar in two). Four patients were treated surgically, with no attempt made at conservative therapy. These surgeries consisted of two C12 posterior arthrodesis procedures with posterior bone graft and wiring, one odontoid screw fixation, and one temporary posterior wiring (which was removed after the synchondrosis healed) in combination with anterior bone grafting at the site of the synchondrosis. Two patients received no initial treatment, and one died before treatment could be given.

Fusion across the synchondrosis was noted in 42 (93\%) of 45 patients treated with external stabilization. In three patients this conservative treatment failed, and they underwent surgical stabilization with posterior $\mathrm{C} 1-2$ arthrodesis. Of the two surviving patients who received no initial treatment within the first 3 months of injury, one healed without intervention and one experienced nonunion requiring posterior C1-2 arthrodesis. All six of the patients who underwent posterior $\mathrm{C} 1-2$ arthrodesis (two as the initial treatment and four after failure of conservative or no treatment) attained fusion. Two C1-2 motion preservation surgeries (one odontoid screw fixation and one temporary posterior wiring with synchondrosis bone grafting) were performed and both were reported to be successful. ${ }^{3}$

\section{Discussion}

Odontoid synchondrosis fracture is one of the more common types of cervical spine fractures in children younger than 7 years of age. The combination of a weak area at the base of the dens with the synchondrosis and the relatively large size of a young child's head makes this area prone to

TABLE 1

Literature review of series reporting children with odontoid synchondrosis fractures

\begin{tabular}{lcc}
\hline \hline \multicolumn{1}{c}{ Authors \& Year } & No. of Patients & Mean Age (yrs) \\
\hline Griffiths, 1972 & 4 & 2.6 \\
Anderson \& D'Alonzo, 1974 & 5 & 4.4 \\
Sherk, et al., 1978 & 11 & 3.0 \\
Mandabach, et al., 1993 & 13 & 3.1 \\
Blauth, et al., 1996 & 3 & 1.8 \\
Odent, et al., 1999 & 15 & 2.2 \\
\hline
\end{tabular}

injury. In many children, this synchondrosis ossifies by the age of 5 to 7 years, but in rare cases it can remain open into adolescence. $^{2}$

The clinical presentation of odontoid synchondrosis fractures can range from neck pain to neurological deficits with SCI. As this combined case series demonstrates, a majority of patients (77\%) will not experience neurological deficits with this fracture. Nevertheless, in children younger than 7 years of age in whom there is an apparent SCI with no apparent fractures according to the initial evaluation on neuroimaging, close evaluation of the odontoid synchondrosis should be undertaken. Odontoid synchondrosis fractures seem to be associated with SCI at the cervicothoracic junction; eight (53\%) of 15 SCIs occurred at this area in this combined series. Odent, et al. ${ }^{8}$ have theorized that SCIs at the cervicothoracic junction are a result of stretch injury to the spinal cord that occurs with this hyperflexion injury.

Radiographic evaluation of the pediatric trauma patient typically starts with plain $\mathrm{x}$-ray films of the cervical spine. Lateral plain x-ray films (Fig. 2A) most often show anterior angulation of the odontoid; $94 \%$ of patients in this combined series had anterior angulation or displacement of the dens. The angulation may be subtle, and it can be overlooked, as demonstrated by the delayed diagnosis in eight of the cases reviewed. The use of CT scanning, especially with sagittal reconstructed images (Fig. 2B) and three-dimensional reconstructions, may be helpful in demonstrat-

TABLE 2

Initial treatment in 52 children with odontoid synchondrosis fractures*

\begin{tabular}{lc}
\hline \multicolumn{1}{c}{ Initial Treatment } & No. of Patients \\
\hline external stabilization & 45 \\
Minerva orthosis & 20 \\
halo vest immobilization & 20 \\
cervical traction & 3 \\
cervical collar & 2 \\
surgical stabilization & 4 \\
pst C1-2 arthrodesis & 2 \\
odontoid screw fixation & 1 \\
pst wiring \& ant bone grafting across synchondrosis, & 1 \\
$\quad$ followed by removal of pst wiring after healing of & \\
$\quad$ odontoid & 2 \\
none & 1 \\
death & 2 \\
\hline
\end{tabular}

$*$ Ant $=$ anterior; pst $=$ posterior. 


\section{Odontoid synchondrosis fractures in children}
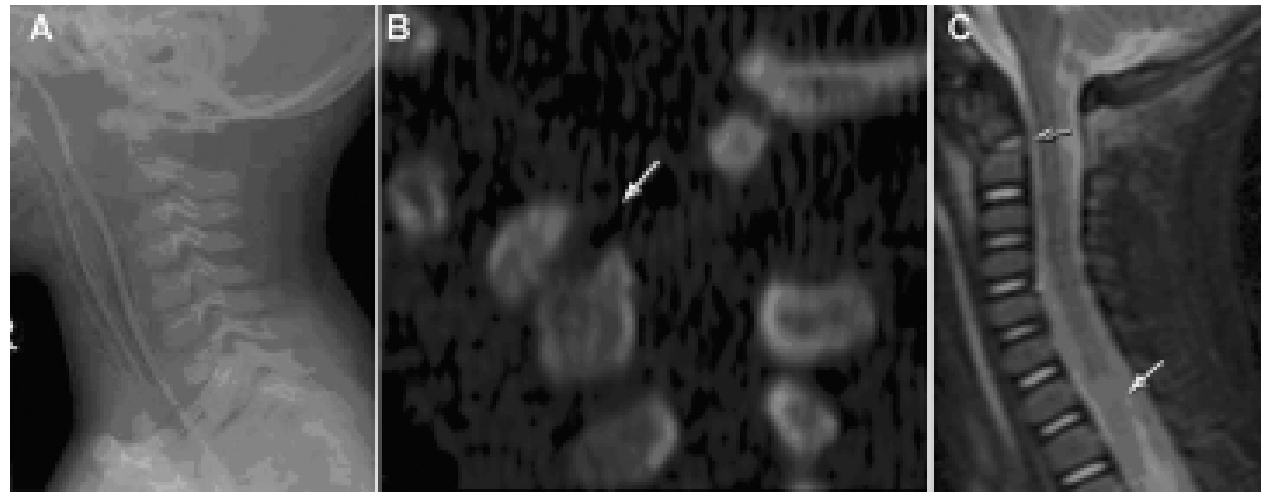

FIG. 2. Radiograph and neuroimages obtained in a 2-year-old boy who was struck by a car and noted to have no motor function in his legs at the accident scene. A: Although the lateral plain x-ray film was initially interpreted to show normal results, there appeared to be anterior angulation of the odontoid on closer inspection. B: Sagittal CT reconstruction revealing angulation of the odontoid and widening of the posterior aspect of the synchondrosis (arrow). C: Sagittal $\mathrm{T}_{2}{ }^{-}$ weighted MR image demonstrating spinal cord edema (white arrow) at the cervicothoracic junction, with high signal at the odontoid synchondrosis (red arrow) and soft tissues anteriorly and posteriorly at C1-2.

ing widening of the synchondrosis. ${ }^{9}$ We have found that MR imaging can help in equivocal cases; a high signal at the synchondrosis and soft-tissue changes at the $\mathrm{C} 1-2$ level provide convincing evidence of a synchondrosis fracture (Fig. 2C).

The initial treatment should be closed reduction and external stabilization, with close radiographic follow up. Rarely, these fractures may be highly unstable, even with external orthosis, and therefore require close follow-up review (Fig. 3 ). One of our patients, who was treated with reduction and halo immobilization, experienced recurrent subluxation of her fracture, which was found on follow-up neuroimages obtained 1 week after initial treatment. Most fractures that are treated for 3 to 6 months in external orthosis will heal (a fusion rate of $93 \%$ was found in this combined series). Insufficient data are available to determine whether one form of external stabilization (Minerva jacket compared with halo or cervical collar) is superior to the others.

Fractures that cannot be maintained in reduction with external stabilization and those that fail to heal after 3 to 6 months should be treated with surgical stabilization. In terms of procedures, motion-sparing surgeries such as odontoid screw fixation or temporary internal stabilization with posterior wiring have been used, but both are challenging and unproven in this young patient population. Posterior C1-2 stabilization with bone grafting and wiring, possibly supplemented with screw fixation (atlantoaxial transarticular or polyaxial screws with rods from C-1 to C-2), has been shown to be an effective way to stabilize the $\mathrm{C} 1-2$ articulation in these young children (Fig. 3C). ${ }^{5}$

\section{Conclusions}

The odontoid synchondrosis in young children is a potential area of weakness in the cervical spine and is prone to fracture when subjected to trauma, especially hyperflexion injuries. These fractures can be difficult to diagnose and require a high level of suspicion. In a child younger than 7
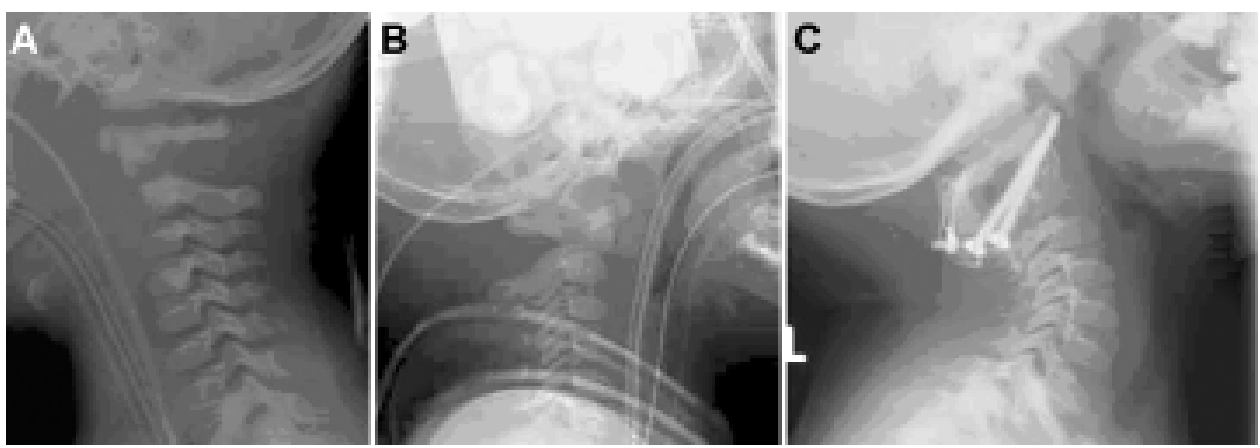

FIG. 3. Lateral x-ray films obtained in a 22-month-old girl who sustained injuries in a motor vehicle accident while restrained in a forward-facing child car seat. No neurological deficits were noted. A: Film obtained at admission, showing anterior angulation of the odontoid process consistent with an odontoid synchondrosis fracture. The patient underwent reduction with the aid of fluoroscopy visualization to confirm good alignment, and was placed in a halo vest orthosis. B: Follow-up x-ray film obtained 1 week after reduction and halo placement, showing recurrent subluxation of the patient's odontoid synchondrosis fracture. She was subsequently treated with surgical stabilization by using posterior C1-2 arthrodesis with atlantoaxial transarticular screws. C: Postoperative films obtained after stabilization; she subsequently attained fusion without complication. 
years of age who presents with neck pain or neurological deficits raising concerns about SCI, an odontoid synchondrosis fracture should be ruled out. Most fractures are initially treated with reduction and external orthosis, which yield excellent fusion rates. Surgical stabilization may be considered for highly unstable fractures that do not maintain alignment despite external orthosis, and in fractures in which nonunion persists despite at least 3 months of conservative treatment.

\section{References}

1. Anderson LD, D'Alonzo RT: Fractures of the odontoid process of the axis. J Bone Joint Surg Am 56:1663-1674, 1974

2. Bailey DK: The normal cervical spine in infants and children. Radiology 59:712-719, 1952

3. Blauth M, Schmidt U, Otte D, et al: Fractures of the odontoid process in small children: biomechanical analysis and report of three cases. Eur Spine J 5:63-70, 1996

4. Connolly B, Emery D, Armstrong D: The odontoid synchondrotic slip: an injury unique to young children. Pediatr Radiol 25:S129-S133, 1995

5. Gluf WM, Brockmeyer DL: Atlantoaxial transarticular screw fixation: a review of surgical indications, fusion rate, complica- tions, and lessons learned in 67 pediatric patients. J Neurosurg Spine 2:164-169, 2005

6. Griffiths SC: Fracture of odontoid process in children. J Pediatr Surg 7:680-683, 1972

7. Mandabach M, Ruge JR, Hahn YS, et al: Pediatric axis fractures: early halo immobilization, management and outcome. Pediatr Neurosurg 19:225-232, 1993

8. Odent T, Langlais J, Glorion C, et al: Fractures of the odontoid process: a report of 15 cases in children younger than 6 years. J Pediatr Orthop 19:51-54, 1999

9. Sherburn EW, Day RA, Kaufman BA, et al: Subdental synchondrosis fracture in children: the value of 3-dimensional computerized tomography. Pediatr Neurosurg 25:256-259, 1996

10. Sherk HH, Nicholson JT, Chung SM: Fractures of the odontoid process in young children. J Bone Joint Surg Am 60:921-924, 1978

11. Sun PP, Poffenbarger GJ, Durham S, et al: Spectrum of occipitoatlantoaxial injury in young children. J Neurosurg (1 Suppl) 93:28-39, 2000

Manuscript received December 15, 2005.

Accepted in final form January 18, 2006.

Address reprint requests to: Douglas Brockmeyer, M.D., Department of Neurosurgery, Primary Children's Medical Center, University of Utah, 100 North Medical Drive, Salt Lake City, Utah 84113. email: douglas.brockmeyer@hsc.utah.edu. 